\title{
ROBUSTNESS OF SOLUTIONS OF LINEAR DIFFERENTIAL EQUATIONS WITH INFINITE DELAY
}

\author{
ANWAR A. AL-BADARNEH (AL-NAYEF) AND RABAA K. MAAITAH \\ Received 22 November 2004; Revised 28 June 2005; Accepted 4 August 2005
}

We use some consequences of the concept of semihyperbolicity of the solution operator to show robustness of solutions of the linear delay differential equation $x^{\prime}(t)=A x(t)+$ $B x(t-r)$ with infinite delay with respect to a small nonlinear perturbation.

Copyright (c) 2006 A. A. Al-Badarneh (Al-Nayef) and R. K. Maaitah. This is an open access article distributed under the Creative Commons Attribution License, which permits unrestricted use, distribution, and reproduction in any medium, provided the original work is properly cited.

\section{Introduction}

The theory of continuous-time dynamical systems began with the study of qualitative properties of the solutions of autonomous ordinary differential equations. It is the principle mathematical technique for describing process that evolves continuously in time. Usually, this qualitative behaviour is described in terms of the solution map of the differential equation defined on the state space. If the iterations of the time-1 map are considered, the resulting system is then called discrete-time dynamical system. Due to round-off error, the approximated behaviour on long time intervals of the dynamical system using numerical techniques is not expected to reflect the behaviour of the original system. The concept of bishadowing provides a practical way of comparing the behaviour of the system and its approximation, see $[6,7,9]$. This concept is usually used to obtain justification of the validity of computation.

In this paper, we use bishadowing to obtain a comparison result of solutions of linear differential equation with infinite delay with respect to its nonlinear perturbation in terms of the solution maps of the two equations defined on the state space $C_{\gamma}$. In Section 2, we give some background material, while Section 3 will be devoted to the main result.

Throughout this paper, $E$ will always denote a Banach space with norm $\|\cdot\|_{E}$. 


\section{Definitions and terminology}

Consider a discrete-time dynamical system generated by iterations $x_{n+1}=f\left(x_{n}\right)$, of a continuous map $f: E \rightarrow E, n \in \mathbb{Z}$, and $n \in \mathbb{Z}^{+}$in case of semidynamical system. For $x_{0} \in E$, let $x_{n}=f^{n}\left(x_{0}\right)$, where $f^{n}$ is the composition of $f$ with itself $n$ times, with $f^{n}=$ $\left(f^{-1}\right)^{|n|}$ if $n<0$ and $f$ is invertible. The forward trajectory through a point $x_{0} \in E$ is the set $\mathrm{O}^{+}\left(x_{0}\right)=\left\{f^{n}\left(x_{0}\right): n \geq 0\right\}$. If $f$ is invertible, then the backward trajectory is the set $0^{-}\left(x_{0}\right)=\left\{f^{n}\left(x_{0}\right): n \leq 0\right\}$, in this case, we define the full trajectory of $x_{0}$ under $f$ to be the set $O\left(x_{0}\right)=\left\{f^{n}\left(x_{0}\right): n \in \mathbb{Z}\right\}$. Similarly, a finite trajectory of the discrete-time dynamical system generated by a map $f$ is a finite sequence $\left\{x_{n}\right\} \subset E$ satisfying $x_{n+1}=f\left(x_{n}\right)$, for $n=-N_{-}, \ldots,-1,0,1, \ldots, N_{+}$where $0 \leq N_{-}, N_{+}<\infty$. A sequence $\left\{y_{n}\right\} \subset E$ is said to be $\gamma$-pseudotrajectory, for $\gamma>0$ if $\left\|y_{n+1}-f\left(y_{n}\right)\right\|_{E} \leq \gamma$, for $n=-N_{-}, \ldots,-1,0,1, \ldots, N_{+}$ where $0 \leq N_{-}, N_{+}<\infty$. A trajectory $\left\{x_{n}\right\}$ is said to $\varepsilon$-shadow a $\gamma$-pseudotrajectory $\left\{y_{n}\right\}$ if $\left\|x_{n}-y_{n}\right\|_{E} \leq \varepsilon$.

2.1. Condensing operators. The Hausdorff measure of noncompactness $\psi(M)$ of a nonempty bounded subset $M$ of $E$ is defined by

$$
\psi(M)=\inf \{r>0: M \text { can be covered by finitely many balls of radius } r\}
$$

Note that $\psi(M)=0$ if and only if $M$ is relatively compact. Some properties of $\psi$ are, see $[5]$ :

(1) if $M_{1} \subseteq M_{2}$, then $\psi\left(M_{1}\right) \leq \psi\left(M_{2}\right)$;

(2) $\psi\left(M_{1}+M_{2}\right) \leq \psi\left(M_{1}\right)+\psi\left(M_{2}\right)$;

(3) $\psi(k M)=|k| \psi(M), k \in \mathbb{R}$.

Let $X \subset E$ and $f: X \rightarrow E$ be continuous, then $f$ is called $\psi$-condensing on $X$ if $\psi(f(M))<$ $\psi(M)$ whenever $M \subset X$ is bounded and not relatively compact. A condensing map $f$ is said to be $\chi$ - $\psi$-contracting for some $0 \leq \chi<1$ if $\psi(f(M)) \leq \chi \psi(M)$. We will call a map $f: E \rightarrow E \delta$-locally $\chi$ - $\psi$-contracting on $K \subset E$ if it is $\chi$ - $\psi$-contracting on each ball $B[x, \delta]$ of radius $\delta$ centered at $x \in K$ with $B[f(x), \delta] \cap K \neq \varnothing$. The $\delta$-locally $\psi$-condensing map is defined in the same way. A continuous map $f: E \rightarrow E$ is completely continuous if it maps bounded sets into a relatively compact sets.

For the following result, see [3].

Lemma 2.1. Let $A: E \rightarrow E$ be a continuous linear operator which is $\chi$ - $\psi$-contracting on $E$ with $0 \leq \chi<1$. Then each $\lambda \in \sigma(A)$ with $|\lambda|>\chi$ is an eigenvalue of $A$ with finite dimensional eigenspace.

2.2. Semihyperbolic mappings. A subset $K \subset E$ is said to be positively invariant with respect to a continuous map $f: E \rightarrow E$ provided that $f(K) \subset K$ and is said to be invariant with respect to $f$ if $f(K)=K$.

The definition of hyperbolicity for diffeomorphisms consists of strong conditions that are very difficult to satisfy in many certain applications. Many generalizations of hyperbolicity were proposed, see, for example [12]. In [7], Diamond et al. gave a new generalization of hyperbolicity called semihyperbolicity. Let $\mathbf{s}=\left(\lambda_{s}, \lambda_{u}, \mu_{s}, \mu_{u}\right)$ be a split, that 
is satisfy $\lambda_{s}<1<\lambda_{u}$ and $\left(1-\lambda_{s}\right)\left(\lambda_{u}-1\right)>\mu_{s} \mu_{u}$ and $K$ a subset of $E$. A Lipschitz map $f: E \rightarrow E$ is said to be s-semihyperbolic on the set $K$ if there exist positive real numbers $k$, $\delta$ and an equivalent norm $\|\cdot\|$ such that for each $x \in K$ there exists a splitting $E=E_{x}^{s} \oplus E_{x}^{u}$ with corresponding projectors $P_{x}^{s}$ and $P_{x}^{u}$ satisfying the following properties:

(SH0) the space $E_{x}^{u}$ is finite dimensional for all $x$ and $\operatorname{dim}\left(E_{x}^{u}\right)=\operatorname{dim}\left(E_{f(x)}^{u}\right)$ if $x, f(x) \in$ $K$;

(SH1) $\sup _{x \in K}\left\{\left\|P_{x}^{s}\right\|,\left\|P_{x}^{u}\right\|\right\} \leq k$;

(SH2) the inequalities

$$
\begin{gathered}
\left\|P_{f(x)}^{s}(f(x+u+v)-f(x+\tilde{u}+v))\right\| \leq \lambda_{s}\|u-\tilde{u}\|, \\
\left\|P_{f(x)}^{s}(f(x+u+v)-f(x+u+\tilde{v}))\right\| \leq \mu_{s}\|v-\tilde{v}\|, \\
\left\|P_{f(x)}^{u}(f(x+u+v)-f(x+\tilde{u}+v))\right\| \leq \mu_{u}\|u-\tilde{u}\|, \\
\left\|P_{f(x)}^{u}(f(x+u+v)-f(x+u+\tilde{v}))\right\| \geq \lambda_{u}\|v-\tilde{v}\|
\end{gathered}
$$

hold for all $x \in K$ with $f(x) \in K$ and all $u, \tilde{u} \in E_{x}^{s}, v, \tilde{v} \in E_{x}^{u}$ such that $\|u\|,\|\tilde{u}\|$, $\|v\|,\|\tilde{v}\| \leq \delta$.

Let

$$
A\left(r_{1}, r_{2}\right)=\left\{z \in \mathbb{C}: r_{1} \leq|z| \leq r_{2}\right\}, \quad A_{0}\left(r_{1}, r_{2}\right)=\left\{z \in \mathbb{C}: r_{1}<|z|<r_{2}\right\}
$$

denote the closed annulus and the open annulus in the complex plane centered at the origin with interior and exterior radii $r_{1}$ and $r_{2}$, respectively. The following is a useful characterization of continuous linear semihyperbolic operators $A: E \rightarrow E$, which was given in [4].

Theorem 2.2. Let $A: E \rightarrow E$ be a continuous linear operator. If

(1) $A$ is $\chi-\psi$-contracting and has no eigenvalues in the annulus $A\left(w_{-}, w_{+}\right)$, where $\chi \leq$ $w_{-}<1<w_{+}$, then $A$ is semihyperbolic on all of $E$ with a split $\left(w_{-}, w_{+}, 0,0\right)$;

(2) $A$ is s-semihyperbolic on the singleton set $\{0\}$ with a split $\mathbf{s}=\left(\lambda_{s}, \lambda_{u}, \mu_{s}, \mu_{u}\right)$, then $A$ is $\lambda_{s}-\psi$-contracting and has no spectral value in the open annulus $A_{0}\left(w_{-}, w_{+}\right)$, where $w_{ \pm}$are defined by

$$
w_{ \pm}=1 \pm \min \left\{1, \frac{1}{2}\left(\lambda_{u}-\lambda_{s}-\sqrt{\left(\lambda_{u}-\lambda_{s}\right)^{2}-4\left(1-\lambda_{s}\right)\left(\lambda_{u}-1\right)+4 \mu_{s} \mu_{u}}\right)\right\}
$$

2.3. Shadowing properties. Shadowing properties are usually used to compare true trajectories of the system with those obtained by numerical methods. The classical Shadowing lemma says that, under certain assumptions on $f$, such as hyperbolicity, for every $\varepsilon>$ 0 there exists a $\gamma>0$ such that each $\gamma$-pseudotrajectory is $\varepsilon$-shadowed by a true trajectory. This kind of shadowing is sometimes called direct shadowing. On the other hand, indirect shadowing is motivated by the question whether every true trajectory can be approximated by some $\gamma$-pseudotrajectory, see [13]. Diamond et al. [8] introduced a composite 
of the two concepts of shadowing in $\mathbb{R}^{d}$, called bishadowing. Let $\operatorname{Tr}(f, K, \gamma)$ denote the set of all finite or infinite $\gamma$-pseudo-trajectories of $f$ that belong to a subset $K \subseteq X$. Since a true trajectory is also a $\gamma=0$ pseudotrajectory, the set of all finite or infinite trajectories which belong to $K$ will be denoted by $\operatorname{Tr}(f, K, 0)$. Note that $\operatorname{Tr}(f, K, 0) \subset \operatorname{Tr}(f, K, \gamma)$. We use the seminorm

$$
\|\varphi-f\|_{\infty}=\sup _{x \in E}\|\varphi(x)-f(x)\|_{E}
$$

to measure the proximity of functions on $E$. We now state the definition of bishadowing in the context of Banach spaces rather than in $\mathbb{R}^{d}$ with comparison maps is $\delta$-locally $\psi$-condensing maps, see [4].

Definition 2.3 [4]. A map $f: E \rightarrow E$ is said to be $\psi$-bishadowing on a subset $K$ of $E$ with positive parameters $\alpha, \beta$, and $\delta$ if for any given finite pseudotrajectory $\mathbf{y}=\left\{y_{n}\right\} \in$ $\operatorname{Tr}(f, K, \gamma)$ with $0 \leq \gamma \leq \beta$ and any $\delta$-locally $\psi$-condensing map $\varphi: E \rightarrow E$ satisfying

$$
\gamma+\|\varphi-f\|_{\infty} \leq \beta
$$

there exists a trajectory $\mathbf{x}=\left\{x_{n}\right\} \in \operatorname{Tr}(\varphi, E, 0)$ such that

$$
\left\|x_{n}-y_{n}\right\|_{E} \leq \alpha\left(\gamma+\|\varphi-f\|_{\infty}\right)
$$

for all $n$ for which $\mathbf{y}$ is defined.

Theorem 2.4 [4]. Let $f: E \rightarrow E$ be a Lipschitz map which is semihyperbolic on a subset $K$ of $E$ with a split $\mathbf{s}=\left(\lambda_{s}, \lambda_{u}, \mu_{s}, \mu_{u}\right)$ and positive constants $k$ and $\delta$. Then it is $\psi$-bishadowing on $K$ for $\delta$-locally $\psi$-condensing comparison map with bishadowing parameters

$$
\begin{gathered}
\alpha(\mathbf{s}, k)=k \frac{\lambda_{u}-\lambda_{s}+\mu_{s}+\mu_{u}}{\left(1-\lambda_{s}\right)\left(\lambda_{u}-1\right)-\mu_{s} \mu_{u}}, \\
\beta(\mathbf{s}, k, \delta)=\delta k^{-1} \frac{\left(1-\lambda_{s}\right)\left(\lambda_{u}-1\right)-\mu_{s} \mu_{u}}{\max \left\{\lambda_{u}-1+\mu_{s}, 1-\lambda_{s}+\mu_{u}\right\}} .
\end{gathered}
$$

A restriction of the concept of $\psi$-bishadowing that uses $\chi$ - $\psi$-contracting comparison maps is given in [4].

Definition $2.5((\alpha, \beta, \chi)$-bishadowing). A map $f: E \rightarrow E$ is said to be $(\alpha, \beta, \chi)$-bishadowing on a subset $K$ of $E$ if for any finite pseudotrajectory $\mathbf{y}=\left\{y_{n}\right\} \in \operatorname{Tr}(f, K, \gamma)$ with $0 \leq \gamma \leq \beta$ and any $\chi$ - $\psi$-contracting map $D: E \rightarrow E$ satisfying

$$
\gamma+\|D\|_{\infty} \leq \beta
$$


there exists a trajectory $\mathbf{x}=\left\{x_{n}\right\} \in \operatorname{Tr}(f+D, E, 0)$ such that

$$
\left\|x_{n}-y_{n}\right\|_{E} \leq \alpha\left(\gamma+\|D\|_{\infty}\right)
$$

for all $n$ for which $\mathbf{y}$ is defined, where $\|D\|_{\infty}=\sup _{x \in E}\|D(x)\|_{E}$.

\section{Main results}

Consider the linear delay differential equation with infinite delay

$$
x^{\prime}(t)=A x(t)+B x(t-r)
$$

where $x(t) \in \mathbb{R}^{d}, A$ and $B$ are $d \times d$ matrices, and $r$ is the time delay, $0 \leq r \leq \infty$. To set up the initial value problem for this equation, an initial function $\varphi$ taken from a specified initial state space needs to be specified on the interval $[-r, 0]$, where $[-r, 0]=(-\infty, 0]$ in case $r=\infty$.

In this paper, we consider the phase space $C_{\gamma}, \gamma>0$, see $[10,11]$, as the state space for (3.1). For any real number $\gamma>0$, define

$$
C_{\gamma}=\left\{\varphi \in C\left((-\infty, 0], \mathbb{R}^{d}\right): \lim _{\theta \rightarrow-\infty} e^{\gamma \theta} \varphi(\theta) \text { exists in } \mathbb{R}^{d}\right\}
$$

with

$$
\|\varphi\|_{C_{\gamma}}=\sup \left\{e^{\gamma \theta}|\varphi(\theta)|:-\infty<\theta \leq 0\right\}, \quad \varphi \in C_{\gamma} .
$$

We will also consider the nonlinear perturbation of (3.1) of the form

$$
y^{\prime}(t)=A y(t)+B y(t-r)+G(y(t), y(t-r)),
$$

where $y(t) \in \mathbb{R}^{d}$ and $G(\cdot, \cdot): C_{\gamma} \times C_{\gamma} \rightarrow \mathbb{R}^{d}$ is continuous, uniformly bounded and locally Lipschitz in its first variable. For each $\varphi \in C_{\gamma}$, (3.4) has a unique solution denoted by $y(t, \varphi, G)$ for $t \geq-r$ that satisfies (3.4) for all $t \geq 0$ and the condition $y(t, \varphi, G)=\varphi(t)$ for $t \in[-r, 0]$. Let $L(G)$ denote the set of all such solutions and $L(0)$ the corresponding set of solutions $x(\cdot, \varphi)$ of the linear equation (3.1). We will also use the notation $x(t)$ and $y(t)$ to denote the solutions in $L(0)$ and $L(G)$, respectively.

Define the solution operator $T(t): C_{\gamma} \rightarrow C_{\gamma}, t \geq 0$ of $(3.1)$ by $T(t) \varphi=x_{t}(\cdot, \varphi)$ and that $T_{G}: C_{\gamma} \rightarrow C_{\gamma}$ of $(3.4)$ by $T_{G}(t) \varphi=y_{t}(\cdot, \varphi, G)$, where the function $x_{t}:[-r, 0] \rightarrow \mathbb{R}^{d}$ is defined by $x_{t}(\theta)=x(t+\theta)$, for $-r \leq \theta \leq 0$, and $y_{t}$ defined similarly. It is well known that $T(t)$ and $T_{G}(t), t \geq 0$, are semigroups of continuous operators. For $\varphi \in C_{\gamma}$, let $\hat{\varphi}=\{\psi \in$ $\left.C_{\gamma}:\|\psi-\varphi\|_{C_{\gamma}}=0\right\}$ be the equivalence class generated by $\varphi$ and $\hat{C}_{\gamma}=\left\{\hat{\varphi}: \varphi \in C_{\gamma}\right\}$ the quotient space. With the norm $\|\hat{\varphi}\|_{\hat{C}_{\gamma}}=\|\varphi\|_{C_{\gamma}}$, the space $\left(\widehat{C}_{\gamma},\|\cdot\|_{\hat{C}_{\gamma}}\right)$ is a Banach space, see [11]. Define operators $\widehat{T}(t)$ and $\widehat{T}_{G}(t)$ on $\widehat{C}_{\gamma}$ induced by $T(t)$ and $T_{G}(t)$, respectively by the formula $\hat{T}(t) \hat{\varphi}=T(t) \varphi$ and $\hat{T}_{G}(t) \hat{\varphi}=T_{G}(t) \varphi$ for $\varphi \in \hat{\varphi}$. Clearly, $\hat{T}(t)$ and $\widehat{T}_{G}(t)$ are semigroups of continuous operators on $\hat{C}_{\gamma}$.

Let $S(t): C_{\gamma} \rightarrow C_{\gamma}, t>0$, and $S(0)=I$ be the solution operator of the equation $x^{\prime}=0$, regarded as a delay equation. It is continuous and linear for each $t \geq 0$. The following representation of the solution operator $T(t)$ can be found in [10]. 
6 Robustness of solutions of infinite delay equation

LEMma 3.1. The solution operator $T(t)$ of (3.1) can be written as $T(t) \varphi=S(t) \varphi+U(t) \varphi$, $t \geq 0$, where $U(t): C_{\gamma} \rightarrow C_{\gamma}$ is completely continuous.

Similar representation can be given for the operators $T_{G}(t), \widehat{T}(t)$, and $\widehat{T}_{G}(t)$ with the corresponding component operators.

Define the characteristic matrix for (3.1) as $\triangle \lambda=\lambda I-A-B e^{-\lambda r}$ and the characteristic equation as

$$
\operatorname{det} \triangle(\lambda)=0 \text {. }
$$

We will say that (3.1) has the saddle point property, see [11], if the space $\widehat{C}_{\gamma}$ can be decomposed as

$$
\widehat{C}_{\gamma}=\widehat{\mathscr{B}}_{s} \oplus \widehat{\mathscr{B}}_{u}
$$

where $\widehat{\mathscr{S}}_{u}$ is finite dimensional and the semigroup $\widehat{T}(t)$ can be defined on $\widehat{\mathscr{B}}_{u}$ for all $t \in(-\infty, \infty)$ and satisfies

$$
\begin{gathered}
\|\hat{T}(t) \hat{\varphi}\|_{\hat{C}_{\gamma}} \leq c e^{v t}\|\hat{\varphi}\|_{\hat{C}_{\gamma}}, \quad t \leq 0, \hat{\varphi} \in \widehat{\mathscr{B}}_{u}, \\
\|\hat{T}(t) \hat{\psi}\|_{\hat{C}_{\gamma}} \leq c e^{-v t}\|\hat{\psi}\|_{\hat{C}_{\gamma}}, \quad t \geq 0, \hat{\psi} \in \widehat{\mathscr{B}}_{s},
\end{gathered}
$$

for some constants $c>0$ and $v>0$.

Theorem 3.2 [11]. Suppose the characteristic equation $\operatorname{det} \triangle(\lambda)=0$ has no roots on the imaginary axis of the complex plane. Then (3.1) has a saddle point property.

Let $T:=T(r): C_{\gamma} \rightarrow C_{\gamma}$ and $T_{G}:=T_{G}(r): C_{\gamma} \rightarrow C_{\gamma}$ be the solution operators of (3.1) and (3.4), respectively, defined by $(T \varphi)(t)=x(t+r, \varphi)$ and $\left(T_{G} \varphi\right)(t)=y(t+r, \varphi, G)$. Note that by Lemma 3.1, we have the following decomposition of $T$ :

$$
T \varphi=S \varphi+U \varphi
$$

where $S:=S(r), U:=U(r)$, and $U: C_{\gamma} \rightarrow C_{\gamma}$ is completely continuous. The operators $S$ and $U$ have the following representations, see [10],

$$
\begin{gathered}
(S \varphi)(t)= \begin{cases}\varphi(t+r)-\varphi(0) & \text { if } t+r<0, \\
0 & \text { if } t+r \geq 0,\end{cases} \\
(U \varphi)(t)= \begin{cases}\varphi(0) & \text { if } t+r<0, \\
\int_{0}^{t+r}[A \varphi(s)+B \varphi(s-r)] d s & \text { if } t+r \geq 0 .\end{cases}
\end{gathered}
$$

The main result of the paper is then the following theorem. 
Theorem 3.3. Let the linear equation (3.1) has the saddle point property on $C_{\gamma}$ with $\gamma>0$ and let $G(u, v)$ be locally Lipschitz in $u$. Then there exists a constant $\zeta>0$ with the following properties:

(a) for each $x(\cdot) \in L(0)$ and for each uniformly bounded $G(u, v)$ there exists a function $y(\cdot) \in L(G)$, satisfying the inequality

$$
|y(t)-x(t)|<\zeta \sup _{u, v}|G(u, v)|, \quad t \geq-r
$$

(b) let $G(u, v)$ be uniformly bounded and $y(\cdot) \in L(G)$. Then there exists a function $x(\cdot) \in$ $L(0)$ satisfying (3.10).

This theorem demonstrates robustness of solutions of the linear equation (3.1) with respect to solutions of the nonlinear equation (3.4), provided that $G$ is "small."

The following lemma is a slight modification of [1, Lemma 3.2], a proof is given in [14], see also $[4,10]$.

Lemma 3.4. A nonzero complex number $\lambda$ is in the point spectrum $P_{\sigma}(\hat{T})$ of $\widehat{T}: \hat{C}_{\gamma} \rightarrow \hat{C}_{\gamma}$, $\gamma>0$, if and only if $\lambda=e^{w r}$, where $w$ is a solution of $\operatorname{det} \triangle(\lambda)=0$.

Consider the closed subspace $C_{\gamma}^{0}$ of $C_{\gamma}$ defined by

$$
C_{\gamma}^{0}=\left\{\varphi \in C_{\gamma}: \varphi(0)=0\right\}
$$

Lemma 3.5. The solution operator $\widehat{T}: \hat{C}_{\gamma}^{0} \rightarrow \widehat{C}_{\gamma}^{0}$ of (3.1) is linear, bounded, and $e^{-\gamma r}-\psi$ contracting.

Proof. The linearity of (3.1) implies that of T. For $\varphi \in C_{\gamma}^{0}$ there exists $x(\cdot):=x(\cdot, \varphi) \in C_{\gamma}^{0}$ such that $T \varphi=x_{r}(\cdot)$. By the decomposition (3.8), $T \varphi=S \varphi+U \varphi$. Since $x(t)$ is a solution of (3.1), then

$$
x^{\prime}(t+r) e^{\gamma(t+r)}=A x(t+r) e^{\gamma(t+r)}+B x(t) e^{\gamma(t+r)}, \quad-r \leq t \leq 0 .
$$

Integrating over the interval $[-r, t]$, where $-r \leq t \leq 0$, we obtain

$$
x(t+r) e^{\gamma(t+r)}=x(0)+\int_{-r}^{t} \gamma x(s+r) e^{\gamma(s+r)} d s+\int_{-r}^{t} A x(s+r) e^{\gamma(s+r)} d s+\int_{-r}^{t} B x(s) e^{\gamma(s+r)} d s .
$$

Since $x(0)=\varphi(0)$, we get

$$
|x(t+r)| e^{\gamma(t+r)} \leq\|\varphi\|_{C_{\gamma}^{0}}+(\gamma+|A|) \int_{-r}^{t}|x(s+r)| e^{\gamma(s+r)} d s+r|B| e^{\gamma r}\|\varphi\|_{C_{\gamma}^{0}} .
$$

By Grownwall's inequality

$$
|x(t+r)| e^{\gamma(t+r)} \leq\left(1+r|B| e^{\gamma r}\right) e^{(\gamma+|A|) r}\|\varphi\|_{C_{\gamma}^{0}}
$$


8 Robustness of solutions of infinite delay equation

that is

$$
\|U \varphi\|_{C_{\gamma}^{0}}=\left\|x_{r}\right\|_{C_{\gamma}^{0}} \leq\left(1+r|B| e^{\gamma r}\right) e^{(\gamma+|A|) r}\|\varphi\|_{C_{\gamma}^{0}}=K_{1}\|\varphi\|_{C_{\gamma}^{0}}
$$

where $K_{1}=\left(1+r|B| e^{\gamma r}\right) e^{|A| r}$.

On the other hand, since $\|S \varphi\|_{C_{\gamma}^{0}}=\sup _{t \leq-r}|\varphi(t+r)| e^{\gamma t}$ and putting $\tau=t+r$ we have

$$
\|S \varphi\|_{C_{\gamma}^{0}}=\sup _{\tau \leq 0}|\varphi(\tau)| e^{\gamma(\tau-r)}=e^{-\gamma r}\|\varphi\|_{C_{\gamma}^{0}}
$$

Combining (3.16) and (3.17) we obtain

$$
\|T \varphi\|_{C_{\gamma}^{0}} \leq\left(K_{1}+e^{-\gamma r}\right)\|\varphi\|_{C_{\gamma}^{0}}
$$

which shows that $T$ is bounded. It remains to show that $T$ is $e^{-\gamma r}-\psi$-contracting.

From (3.1), we have

$$
\begin{aligned}
\left|x^{\prime}(t+r)\right| e^{\gamma(t+r)} \leq & |A||x(t+r)| e^{\gamma(t+r)}+|B||x(t)| e^{\gamma(t+r)} \\
& +|A||x(0)| e^{\gamma(t+r)}+|B||x(-r)| e^{\gamma(t+r)} .
\end{aligned}
$$

Since $x(t)=\varphi(t), t \in[-r, 0]$, we obtain

$$
\begin{aligned}
\left|x^{\prime}(t+r)\right| e^{\gamma(t+r)}= & |A| e^{\gamma r} \sup _{-r \leq t \leq 0}\left|x_{r}(t)\right| e^{\gamma t}+|B| e^{\gamma r} \sup _{-r \leq t \leq 0}|\varphi(t)| e^{\gamma t} \\
& +|A| e^{\gamma r} \sup _{-r \leq t \leq 0}|\varphi(0)| e^{\gamma t}+|B| e^{\gamma r} \sup _{-r \leq t \leq 0}|\varphi(-r)| e^{\gamma t} \\
\leq & |A| e^{\gamma r}\left\|x_{r}\right\|_{C_{\gamma}^{0}}+|B| e^{\gamma r}\|\varphi\|_{C_{\gamma}^{0}}+|A| e^{\gamma r}\|\varphi\|_{C_{\gamma}^{0}}+|B| e^{\gamma r}\|\varphi\|_{C_{\gamma}^{0}} \\
\leq & \left(|A| K_{1}+|A|+2|B|\right) e^{\gamma r}\|\varphi\|_{C_{\gamma}^{0} .}
\end{aligned}
$$

Thus

$$
\left\|U^{\prime} \varphi\right\|_{C_{\gamma}^{0}}=\sup _{-r \leq t \leq 0}\left|\left(U^{\prime} \varphi\right)(t)\right| e^{\gamma t}=\sup _{-r \leq t \leq 0}\left|x_{r}^{\prime}(t)\right| e^{\gamma t} \leq K_{2}\|\varphi\|_{C_{\gamma}^{0}},
$$

where $K_{2}=|A| K_{1}+|A|+2|B|$.

Let $\mathcal{M}=\left\{\varphi \in C_{\gamma}^{0}:\|\varphi\|_{C_{\gamma}^{0}} \leq 1\right\}$. For each $\varphi \in \mathcal{M}$ and by virtue of the relations (3.16) and (3.21), the functions $U^{\prime} \varphi$ and $U \varphi$ are uniformly bounded. The Arzelà theorem implies that the set $U M$ is precompact, and hence $\psi(U M)=0$. Finally, by the relation (3.17),

$$
\psi(T . M) \leq \psi(S M)+\psi(U M) \leq e^{-\gamma r}
$$

This shows that $T$ is $e^{-\gamma r}-\psi$-contracting.

Lemma 3.6. If (3.1) has the saddle point property, then the solution operator $\widehat{T}: \hat{C}_{\gamma}^{0} \rightarrow \hat{C}_{\gamma}^{0}$ is semihyperbolic. 
Proof. Since the solution operator $\widehat{T}: \hat{C}_{\gamma}^{0} \rightarrow \hat{C}_{\gamma}^{0}$ of (3.1) is linear, bounded, and $e^{-\gamma r}$ contracting operator, then each $\lambda \in \sigma(\widehat{T})$ with $|\lambda|>e^{-\gamma r}$ is an eigenvalue of $\hat{T}$, that is, belongs to the point spectrum $P_{\sigma}(\hat{T})$ of $\hat{T}$, with finite dimensional eigenspace, see [3]. Define the sets $E_{1}$ and $E_{2}$ by

$$
E_{1}=\left\{\lambda \in P_{\sigma}(\widehat{T}): e^{-\gamma r}<|\lambda|<1\right\}, \quad E_{2}=\left\{\lambda \in P_{\sigma}(\widehat{T}):|\lambda|>1\right\}
$$

which are finite sets. Let $w_{-}=\max \left\{|\lambda|: \lambda \in E_{1}\right\}$ and $w_{+}=\min \left\{|\lambda|: \lambda \in E_{2}\right\}$. Therefore, there are no eigenvalues in the annulus $A\left(w_{-}, w_{+}\right)$. Since, by Lemma 3.4, the set of nonzero eigenvalues of the linear operator $\hat{T}$ coincides with the set of complex numbers $\lambda=e^{w r}$, where $w$ is a solution of the characteristic equation, then, by Theorem 2.2, $\widehat{T}$ is semihyperbolic on $\hat{C}_{\gamma}^{0}$ with the split $\left(w_{-}, w_{+}, 0,0\right)$.

The following lemma follows from Theorem 2.2.

LEMma 3.7. If (3.1) has the saddle point property, then the solution operator $\widehat{T}: \hat{C}_{\gamma}^{0} \rightarrow \hat{C}_{\gamma}^{0}$ is $(\alpha, \beta, \chi)$-bishadowing on $\hat{C}_{\gamma}^{0}$ with $\alpha$ and $\beta$ given by the relations (2.8) and for any $\chi<1-w_{-}$. In particular, if

$$
\gamma>\frac{1}{r} \ln \left(\frac{1}{1-w_{-}}\right),
$$

then the solution operator $\widehat{T}$ is $\left(\alpha, \beta, e^{-\gamma r}\right)$-bishadowing on $\hat{C}_{\gamma}^{0}$ with $\alpha$ and $\beta$ given by (2.8).

Lemma 3.8. The solution operator $\widehat{T}_{G}: \hat{C}_{\gamma}^{0} \rightarrow \hat{C}_{\gamma}^{0}$ of the nonlinear equation (3.4) is continuous and $e^{-\gamma r}-\psi$-contracting.

Proof. By (3.8), write the operator $T_{G}$ as $T_{G} \varphi=U_{G} \varphi+S_{G} \varphi$, for $\varphi \in C_{\gamma}^{0}$, where $U_{G}:=$ $U_{G}(r)$ and $S_{G}:=S_{G}(r)$. Let $\varphi, \phi \in C_{\gamma}^{0}$ and consider $Y(t)=y(t, \varphi+\phi, G)-y(t, \phi, G), t \geq$ 0 , where $y(t, \varphi+\phi, G)$ and $y(t, \phi, G)$ are the solutions of the nonlinear equation (3.4) through the initial functions $\varphi+\phi$ and $\phi$, respectively. Note that $Y(t)$ satisfies the equation

$$
\begin{aligned}
Y(t)= & A Y(t)+B Y(t-r)+G(y(t, \varphi+\phi, G), y(t-r, \varphi+\phi, G)) \\
& -G(y(t, \phi, G), y(t-r, \phi, G))
\end{aligned}
$$

with initial condition $Y(t)=\varphi(t), t \in[-r, 0]$. Calculations proceed in a similar way as in the proof of Theorem 3.3, so we only give the main steps. Denote by $L_{G}$ the Lipschitz constant of $G$ in its first variable, then

$$
|Y(t+r)| e^{\gamma(t+r)} \leq\left(1+r|B| e^{\gamma r}+r L_{G} e^{\gamma r}\right)\|\varphi\|_{C_{\gamma}^{0}}+(\gamma+|A|) \int_{-r}^{t}|Y(s+r)| e^{\gamma(s+r)} d s .
$$

Using Grownwall's inequality, $|Y(t+r)| e^{\gamma(t+r)} \leq\left(1+r|B| e^{\gamma r}+r L_{G} e^{\gamma r}\right) e^{(\gamma+|A|) r}\|\varphi\|_{C_{\gamma}^{0}}$, and hence

$$
\left\|U_{G}\right\|_{C_{\gamma}^{0}}=\left\|Y_{r}\right\|_{C_{\gamma}^{0}}=\sup _{-r \leq t \leq 0}\left|Y_{r}(t)\right| e^{\gamma t} \leq K_{3}\|\varphi\|_{C_{\gamma}^{0}}
$$


where $K_{3}=\left(1+r|B| e^{\gamma r}+r L_{G} e^{\gamma r}\right) e^{|A| r}$. On the other hand, for $t \geq-r$, we have

$$
\begin{aligned}
\left|(U \varphi)^{\prime}(t)\right| \leq & |A||Y(t+r)|+|B||Y(t)|+|A||Y(0)|+|B||Y(-r)| \\
& +|G(y(t+r, \varphi+\phi, G), y(t, \varphi+\phi, G))-G(y(t+r, \phi, G), y(t, \phi, G))| \\
\leq & |A||Y(t+r)|+|B||\varphi(t)|+|A||\varphi(0)|+|B||\varphi(-r)|+L_{G}|\varphi(t)| .
\end{aligned}
$$

So

$$
\left\|\left(U_{G} \varphi\right)^{\prime}\right\|_{C_{\gamma}^{0}}=\left\|Y_{r}^{\prime}\right\|_{C_{\gamma}^{0}}=\sup _{-r \leq t \leq 0}\left|Y_{r}^{\prime}(t)\right| e^{\gamma t} \leq\left(|A| K_{3}+2|B|+|A|+L_{G}\right)\|\varphi\|_{C_{\gamma}^{0}} .
$$

From the estimations (3.27) and (3.29), the functions $\left(U_{G} \varphi\right)^{\prime}$ and $U_{G} \varphi$ are uniformly bounded. By Arzelà theorem, $U_{G} \mathcal{M}$ is precompact, where $\mathcal{M}=\left\{\varphi \in C_{\gamma}^{0}:\|\varphi\|_{C_{\gamma}^{0}} \leq 1\right\}$, and hence $\psi\left(U_{G} \mathcal{M}\right)=0$. Since $\psi\left(S_{G} \mathcal{M}\right)=\psi(S \mathcal{M}) \leq e^{-\gamma r}$, we get

$$
\psi\left(T_{G} M\right) \leq \psi\left(S_{G} M\right)+\psi\left(U_{G} M\right) \leq e^{-\gamma r} .
$$

This proves that $T_{G}$ is $e^{-\gamma r}-\psi$-contracting, which ends the proof of the lemma.

Lemma 3.9. There exists a positive constant $c_{1}$ such that

$$
\left\|\widehat{T}_{G} \hat{\varphi}-\hat{T} \hat{\varphi}\right\|_{\hat{C}_{\gamma}^{0}} \leq c_{1} \sup _{u, v \in \mathbb{R}^{d}}|G(u, v)|
$$

for $\hat{\varphi} \in \hat{C}_{\gamma}^{0}, t \geq-r$.

Proof. From (3.1) and (3.4) and for $t \geq-r$, we have

$$
\begin{aligned}
\left|\left(T_{G} \varphi\right)(t)-(T \varphi)(t)\right|= & \left|\left(U_{G} \varphi\right)(t)-(U \varphi)(t)\right| \\
\leq & |A| \int_{-r}^{t}\left|y_{r}(s)-x_{r}(s)\right| d s+|B| \int_{-r}^{t}|y(s)-x(s)| d s \\
& +\int_{-r}^{t}|G(u, v)| d s \\
\leq & |A| \int_{-r}^{t}\left|\left(T_{G} \varphi\right)(s)-(T \varphi)(s)\right| d s+r \sup _{u, v \in \mathbb{R}^{d}}|G(u, v)| .
\end{aligned}
$$

Thus, by Grownwall's inequality

$$
\left|\left(T_{G} \varphi\right)(t)-(T \varphi)(t)\right| \leq r e^{|A| r} \sup _{u, v \in \mathbb{R}^{d}}|G(u, v)| .
$$

That is

$$
\left\|T_{G} \varphi-T \varphi\right\|_{C_{\gamma}^{0}} \leq c_{1} \sup _{u, v \in \mathbb{R}^{d}}|G(u, v)|
$$

where $c_{1}=r e^{|A| r}$. Relation (3.31) then follows from relation (3.34), and the lemma is proved. 
Theorem 3.10. Let (3.1) have the saddle point property and let the function G be uniformly bounded and satisfying

$$
\sup _{u, v \in \mathbb{R}^{d}}|G(u, v)| \leq \frac{1}{r} e^{-(|A|+\gamma) r} .
$$

Then there exists a constant $c_{2}>0$ with the following properties:

(a) for each trajectory

$$
\mathbf{x}=\left\{x_{0}, x_{1}, \ldots\right\}
$$

of the solution operator $\widehat{T}$, there exists a trajectory

$$
\mathbf{y}^{G}=\left\{y_{0}^{G}, y_{1}^{G}, \ldots\right\}
$$

of the solution operator $\widehat{T}_{G}$ with

$$
\left\|x_{n}-y_{n}^{G}\right\|_{C_{\gamma}^{0}} \leq c_{2} \sup _{u, v \in \mathbb{R}^{d}}|G(u, v)|, \quad n=0,1, \ldots, N
$$

(b) for each trajectory (3.37) of the solution operator $\widehat{T}_{G}$ and for each positive integer $N$, there exists a trajectory (3.36) of the solution operator $\hat{T}$ satisfying the relation (3.38).

Proof. Note that by Lemmas 3.5 and 3.8, the solution operators $\widehat{T}$ and $\widehat{T}_{G}$ are both continuous and $e^{-\gamma r}-\psi$-contracting operators. By Lemma 3.6, the operator $\hat{T}$ is semihyperbolic with $\lambda_{s}=w_{-}, \lambda_{u}=w_{+}$, and $\mu_{s}=\mu_{u}=0$ and hence by Lemma 3.7 is $\left(\alpha, \beta, e^{-\gamma r}\right)$ bishadowing with the corresponding values of $\alpha$ and $\beta$. By Lemma 3.9 there exists a constant $c_{1}>0$ such that

$$
\left\|\widehat{T}_{G} \varphi-\widehat{T} \varphi\right\|_{C_{\gamma}^{0}} \leq c_{1} \sup _{u, v \in \mathbb{R}^{d}}|G(u, v)|
$$

It follows from the condition (3.35) that the operator $\widehat{T}_{G}-\widehat{T}$ is $e^{-\gamma r}-\psi$-contracting on $\hat{C}_{\gamma}^{0}$. We now apply Lemma 3.6 with $f=\widehat{T}$ and $D=\widehat{T}_{G}-\widehat{T}$. For any finite initial segment of the given trajectory $\mathbf{x}=\left\{x_{0}, x_{1}, \ldots\right\}$ of $\widehat{T}$, that is, $\mathbf{x} \in \operatorname{Tr}\left(\widehat{T}, \hat{C}_{\gamma}^{0}, \gamma_{1}\right)$ with $\gamma_{1}=0$ and if

$$
\sup _{\hat{\varphi} \in \hat{C}_{\gamma}^{0}}\left\|\widehat{T}_{G} \hat{\varphi}-\hat{T} \hat{\varphi}\right\|_{\hat{C}_{\gamma}^{0}} \leq \beta
$$

there exists a finite initial segment of the trajectory $\mathbf{y}=\left\{y_{0}^{G}, y_{1}^{G}, \ldots\right\} \in \operatorname{Tr}\left(\widehat{T}_{G}, \hat{C}_{\gamma}^{0}, 0\right)$ such that

$$
\left\|x_{n}-y_{n}^{G}\right\|_{\hat{C}_{\gamma}^{0}} \leq \alpha\left(\gamma_{1}+\sup _{u, v \in \mathbb{R}^{d}}\left\|\widehat{T}_{G} \hat{\varphi}-\hat{T} \hat{\varphi}\right\|_{\hat{C}_{\gamma}^{0}}\right) \leq \alpha c_{1} \sup _{u, v \in \mathbb{R}^{d}}|G(u, v)|=c_{2} \sup _{u, v \in \mathbb{R}^{d}}|G(u, v)|,
$$

where $c_{2}=\alpha c_{1}$. 
Conversely, let $\mathbf{x}=\left\{x_{0}, x_{1}, \ldots, x_{N}\right\} \in \operatorname{Tr}\left(\widehat{T}_{G}, \widehat{C}_{\gamma}^{0}, 0\right)$ be a true trajectory of $\widehat{T}_{G}$, then, for $n=0,1, \ldots, N$,

$$
\begin{aligned}
\left\|x_{n+1}-\hat{T} x_{n}\right\|_{\hat{C}_{\gamma}^{0}} & \leq\left\|x_{n+1}-\widehat{T}_{G} x_{n}\right\|_{\hat{C}_{\gamma}^{0}}+\left\|\widehat{T}_{G} x_{n}-\widehat{T} x_{n}\right\|_{\hat{C}_{\gamma}^{0}} \\
& =\left\|\widehat{T}_{G} x_{n}-\widehat{T} x_{n}\right\|_{\hat{C}_{\gamma}^{0}} \leq c_{1} \sup _{u, v \in \mathbb{R}^{d}}|G(u, v)| .
\end{aligned}
$$

This means that $\mathbf{x} \in \operatorname{Tr}\left(T, C_{\gamma}^{0}, \gamma_{2}\right)$, where $\gamma_{2}=c_{1} \sup _{u, v \in \mathbb{R}^{d}}|G(u, v)|$. Now by taking $D=f$, there exists a trajectory $\mathbf{y}^{G}=\left\{y_{0}^{G}, y_{1}^{G}, \ldots, y_{N}^{G}\right\} \in \operatorname{Tr}\left(\widehat{T}, \widehat{C}_{\gamma}^{0}, \gamma_{1}\right)$ such that

$$
\left\|x_{n}-y_{n}\right\|_{\hat{C}_{\gamma}^{0}} \leq \alpha\left(\gamma_{1}+\|\hat{T}-\widehat{T}\|_{\hat{C}_{\gamma}^{0}}\right)=\alpha \gamma_{1}=\alpha c_{1} \sup _{u, v \in \mathbb{R}^{d}}|G(u, v)|=c_{2} \sup _{u, v \in \mathbb{R}^{d}}|G(u, v)|
$$

The proof is completed.

Proof of Theorem 3.3. Recall that the iterates of the linear solution operator $\hat{T}$ are given by

$$
\left(\widehat{T}^{n} \hat{\varphi}\right)=x(t+n r, \hat{\varphi}),
$$

for $-r \leq t \leq 0$ and $n=0,1, \ldots$. So the proof of Theorem 3.3 is a consequence of Theorem 3.10 .

Remark 3.11. The bishadowing result of Theorem 2.4 is valid only for finite trajectories and was extended for infinite trajectories in [2]. Consequently, the result of Theorem 3.10 is also valid for infinite trajectories. Thus by Theorem 3.3 we obtain the robustness of solutions of (3.1) with respect to solutions of the nonlinear equation (3.4).

\section{References}

[1] A. A. Al-Nayef, Semi-hyperbolic mappings in Banach spaces, Ph.D. thesis, Deakin University, Australia, 1997.

[2] _ Bi-shadowing of infinite trajectories for difference equations in Banach spaces, Journal of Difference Equations and Applications 7 (2001), no. 4, 577-585.

[3] - On the spectrum of $\psi$-contracting operators, Journal of Applied Mathematics and Stochastic Analysis 14 (2001), no. 3, 303-308.

[4] A. A. Al-Nayef, P. E. Kloeden, and A. V. Pokrovskii, Semi-hyperbolic mappings, condensing operators, and neutral delay equations, Journal of Differential Equations 137 (1997), no. 2, 320-339.

[5] K. Deimling, Nonlinear Functional Analysis, Springer, Berlin, 1985.

[6] P. Diamond, P. E. Kloeden, V. S. Kozyakin, and A. V. Pokrovskii, Robustness of the observable behavior of semihyperbolic dynamical systems, Avtomatika i Telemekhanika (1995), no. 11, 148159 , Special issue dedicated to M. Krasnosel'skii's 75 anniversary.

[7] Semihyperbolic mappings, Journal of Nonlinear Science 5 (1995), no. 5, 419-431.

[8] P. Diamond, P. E. Kloeden, and A. V. Pokrovskii, Shadowing and approximation in dynamical systems, Miniconference on Analysis and Applications (Brisbane, 1993) (G. Martin and H. B. Thompson, eds.), Proc. Centre Math. Appl. Austral. Nat. Univ., vol. 33, Austral. Nat. Univ., Canberra, 1994, pp. 47-60.

[9] P. Diamond, V. S. Kozyakin, P. E. Kloeden, and A. V. Pokrovskii, Computer robustness of semihyperbolic mappings, Random \& Computational Dynamics 3 (1995), no. 1-2, 57-70. 
[10] J. K. Hale and S. M. Verduyn Lunel, Introduction to Functional-Differential Equations, Applied Mathematical Sciences, vol. 99, Springer, New York, 1993.

[11] Y. Hino, S. Murakami, and T. Naito, Functional-Differential Equations with Infinite Delay, Lecture Notes in Mathematics, vol. 1473, Springer, Berlin, 1991.

[12] B. Lani-Wayda, Hyperbolic Sets, Shadowing and Persistence for Noninvertible Mappings in Banach Spaces, Pitman Research Notes in Mathematics Series, vol. 334, Longman, Harlow, 1995.

[13] S. Yu. Pilyugin, The Space of Dynamical Systems with the $C^{0}$-Topology, Lecture Notes in Mathematics, vol. 1571, Springer, Berlin, 1994.

[14] M. K. Rabaa, Semi-hyperbolicity and differential equations with infinite delay, Master's thesis, Mu’tah University, Jordan, 2003.

Anwar A. Al-Badarneh (Al-Nayef): Department of Mathematics and Statistics, Mu'tah University, 61710 Mu’tah, Jordan

E-mail addresses: anwar@mutah.edu.jo; anwar.a@qu.edu.qa

Rabaa K. Maaitah: Department of Mathematics and Statistics, Mu'tah University,

61710 Mu'tah, Jordan

E-mail address: mnar792000@yahoo.com 


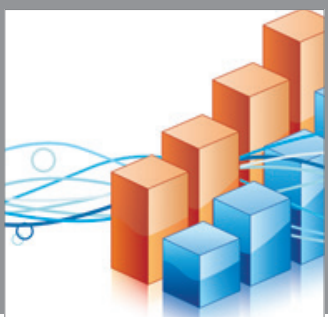

Advances in

Operations Research

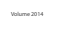

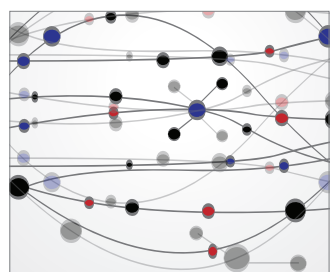

\section{The Scientific} World Journal
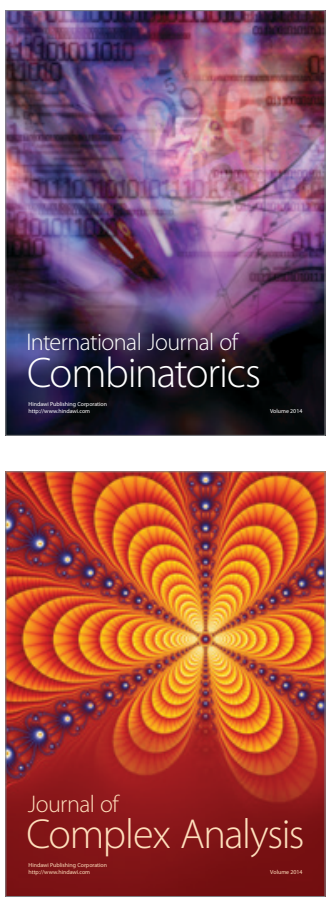

International Journal of

Mathematics and

Mathematical

Sciences
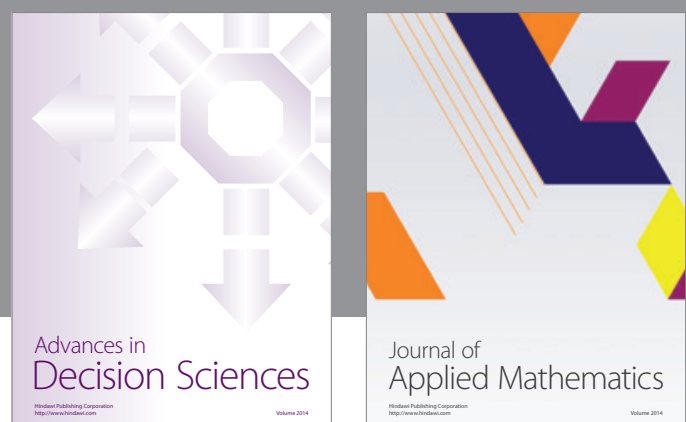

Journal of

Applied Mathematics
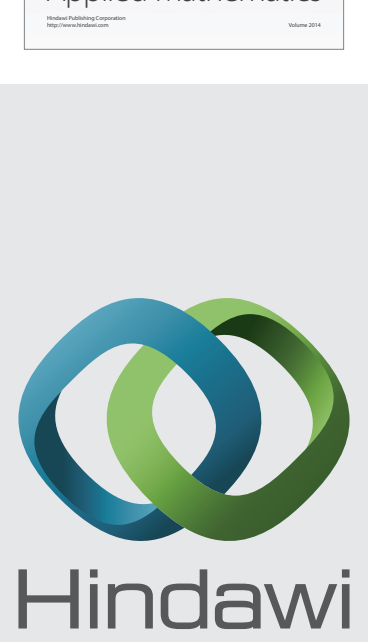

Submit your manuscripts at http://www.hindawi.com
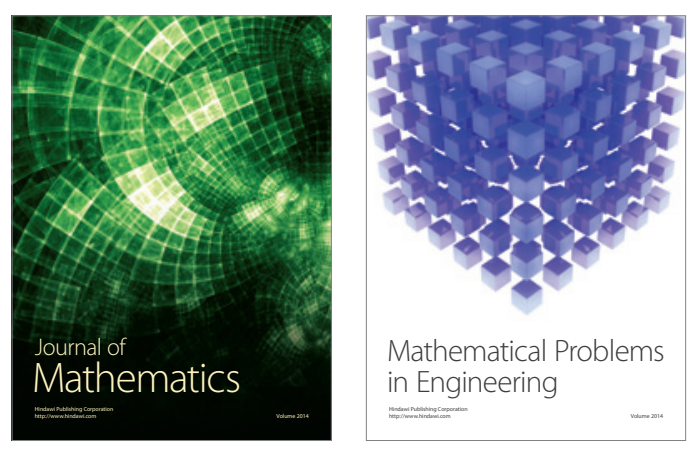

Mathematical Problems in Engineering
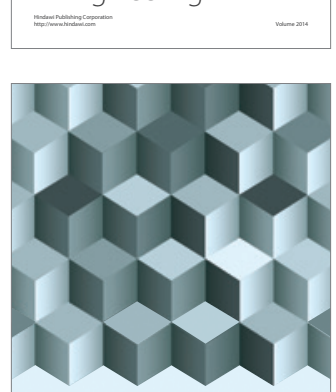

Journal of

Function Spaces
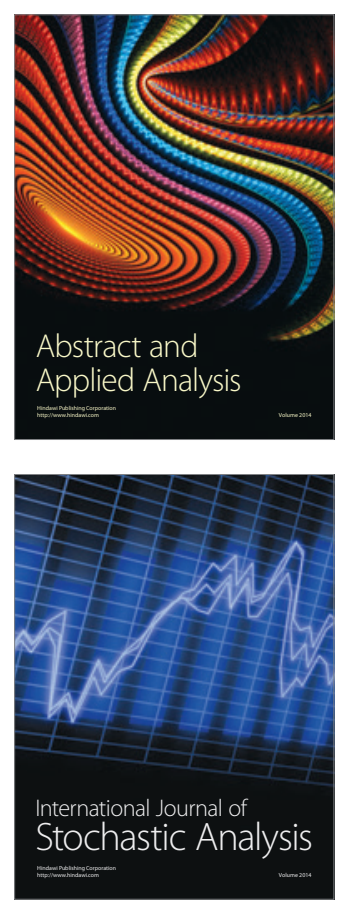

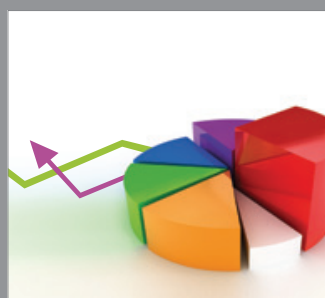

ournal of

Probability and Statistics

Promensencen
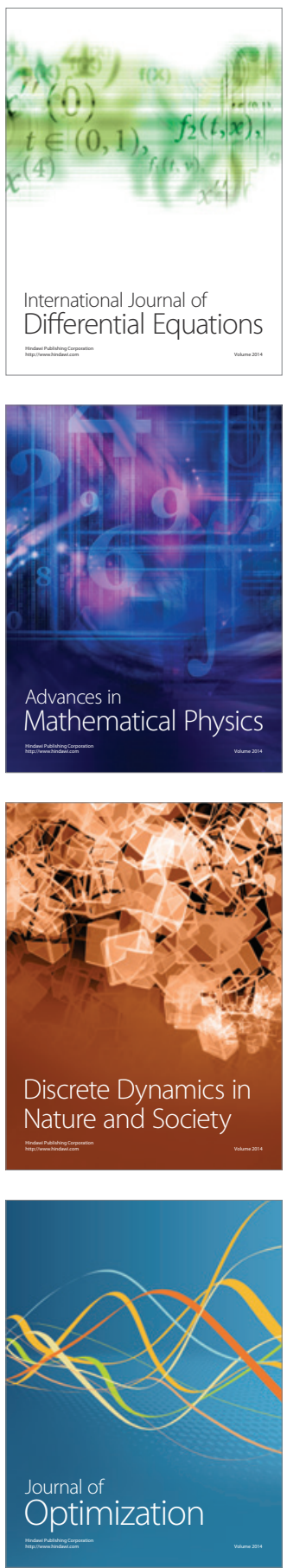